\title{
Competition in Secondary Spectrum Markets: Price War or Market Sharing?
}

\author{
Emir Kavurmacioglu \\ Division of Systems Engineering \\ Boston University \\ Boston, MA 02215 \\ emir@bu.edu
}

\author{
Murat Alanyali \\ Department of Electrical \\ and Computer Engineering \\ Boston University \\ Boston, MA 02215 \\ alanyali@bu.edu
}

\author{
David Starobinski \\ Division of Systems Engineering \\ Boston University \\ Boston, MA 02215 \\ staro@bu.edu
}

\begin{abstract}
Recent initiatives allow cellular providers to offer spot service of their licensed spectrum, paving the way to dynamic secondary spectrum markets. This paper characterizes market outcomes when multiple providers are drawn into competition for secondary demand. We study a game-theoretic model in which each provider aims to enhance its revenue by opportunistically serving secondary demand, while also serving dedicated primary demand. The secondary demand is a function of the price being charged. We consider two philosophies for sharing spectrum between primary and secondary demand: In coordinated access, spectrum providers have the option to decline a secondary access request if that helps enhance their revenue. We explicitly characterize a break-even price such that profitability of secondary access provision is guaranteed if secondary access is priced above the breakeven price, regardless of the volume of secondary demand. Consequently, we establish that competition among providers that employ coordinated access leads to a price war. In particular market sharing above the break-even price is not an equilibrium outcome. This conclusion is valid for arbitrary secondary-demand functions. While the demand function does not play a part in determining the winner, it does affect the price of secondary access as exercised by the winning provider. In uncoordinated access, primary and secondary users share spectrum on equal basis, akin to the sharing modality of ISM bands. We demonstrate that market equilibrium in an uncoordinated access setting can be fundamentally different as it opens up the possibility of providers sharing the market at higher prices.
\end{abstract}

\section{INTRODUCTION}

Recent initiatives by governmental agencies extend the reach of spectrum management policies that license holders (e.g., network providers) are entitled to pursue $[4,7$, 9-11,26]. In particular, the FCC introduced a new spectrum access policy model known as Private Commons to support fast time-scale spectrum transactions $[1,8]$. Under this model, ownership of spectrum remains with the license holder providing service to its primary users, but the provider may also provide spectrum access to secondary users for a fee.
As pointed out in FCC's report on secondary spectrum markets, control of secondary access in private commons can be implemented in several different ways [1]. In particular, access to the spectrum by secondary users may be coordinated by the provider, via signals that determine when or how such access is allowed [8]. A notable coordinated policy is the so-called threshold (reservation) policy, whereby secondary spectrum access is permitted as long as the number of channels occupied in a given spectrum band is below a certain threshold. Theoretical properties of the threshold policy, including optimality in certain settings, have been extensively studied in the literature $(c f .[22,28-30,34]$ and references therein). Access to a band may also be uncoordinated, in which case primary and secondary users share access to the band on an equal basis, in a way similar to ISM bands [8].

Since cellular networks are generally over-provisioned to cope with short-term spikes in their loads, through private commons it might be possible to increase spectrum utilization. For instance, a measurement based study of close to 20,000 GSM base stations deployed in Germany indicates that the majority of base station in crowded areas, such as city centers, remain underloaded at all times [27]. Another study conducted in the Commonwealth of Virginia indicates that the US market is no exception to the case with maximum network occupancy levels around 45\% [3]. The results of these studies point at the possibility of increasing network revenues through secondary service provisions.

Realizing this potential entails a number of challenges for a spectrum provider. One such challenge concerns pricing of secondary spectrum access in the face of uncertainty of demand response to the advertised price: Providing secondary access at a charge returns an immediate revenue for the provider, but it also incurs an opportunity cost due to lost primary revenue because spectrum is fundamentally a finite resource. The balance 


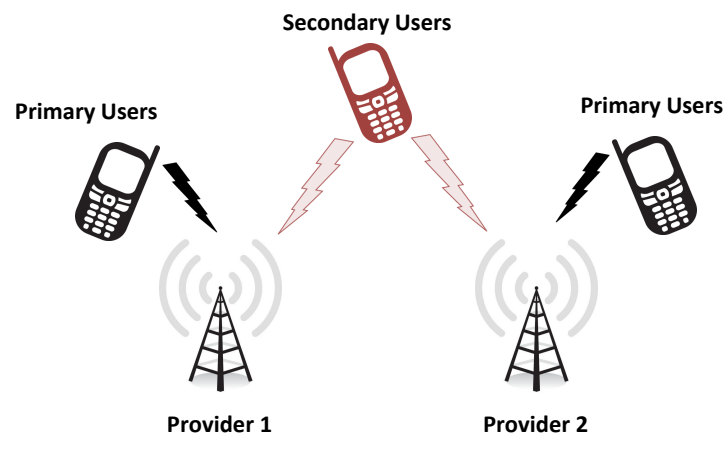

Fig. 1. Illustration of provider competition over the secondary users in a private commons setting

between these two effects determines profitability of secondary spectrum provision, and it may possibly depend not only on the secondary price but also on the secondary demand. The relationship between secondary price and demand, however, is difficult to characterize explicitly and it may also be time-varying.

This issue is further aggravated in competitive situations in which multiple spectrum providers compete for the same pool of secondary demand. In such situations a provider may opt to beat the price of competitors thereby winning entire secondary market, or may opt to match competitors' price thereby serving part of the market but at a higher price. It is not readily clear which alternative is favorable, especially under the alluded uncertainty in the price-demand relationship.

In this paper we seek to analyze the outcomes of a price competition between multiple providers in private commons, as illustrated in Fig I. We consider a game theoretic setting and identify equilibrium prices in term of Nash equilibria. In profit calculations we adopt a model that explicitly captures the random nature of spectrum access requests of both primary and secondary users. Our conclusions make no assumptions on the secondary demand function, and therefore they hold for arbitrary price-demand relations.

Next, we list the main contributions of our paper. Our first contribution is to characterize and then prove the existence of a break-even price for each provider. This break-even price is the lower limit to the price values for which a provider's profitability is guaranteed. The paper explicitly characterizes the break-even price, which is independent of the parameters of other providers and possesses the fundamental property of being insensitive to the specific shape of the demand function of secondary users. The analysis further reveals that the break-even price directly relates to the fraction of lost primary users (in the absence of secondary users), which can be expressed using the well-studied Erlang-B function. The break-even price therefore inherits all the mathematical properties of that function.

We point out that a revenue-maximizing price does depend on the shape of the secondary demand function, and may be significantly higher than the break-even price of each of the competing providers. In contrast to the break-even price, however, revenue-maximizing prices cannot be identified without explicit knowledge of the secondary demand function.

Our next contribution is to show that, under an optimal coordinated access policy, market sharing between providers is not an equilibrium outcome. Thus, a provider that employs optimal coordinated access opts to beat the price of its competitors, leading to a price war. Furthermore this property holds irrespective of the specific relation between the secondary price and the secondary demand. We formally establish the dominating strategy of each provider and list all possible market outcomes (i.e., Nash equilibria), which this price war can lead to. We demonstrate that the provider with the lowest break-even price wins the market. If multiple providers have the same break-even price, they are coerced into an equilibrium in which no provider makes a profit.

As our last contribution, we show that market equilibria under uncoordinated secondary access may be drastically different than those under coordinated access: Through a numerical study, we illustrate that depending on the shape of the secondary demand function, market equilibria may reflect a situation wherein providers share the market by matching each other's price strictly above their respective break-even prices. This outcome may entail negative implications on affordable availability of spectrum and appears to warrant further study.

The rest of the paper is organized as follows. In Section II, we survey previous work. In Section III, we introduce our model of the competition in private commons. Afterwards, in Section IV, we construct the optimal coordinated access policy and derive the breakeven price for each provider. Then, in Section V, we analyze the multi-provider spectrum pricing game arising when each provider implements the optimal coordinated access policy. We numerically contrast these results to the uncoordinated access case in Section VI. We conclude the paper in Section VII.

For the interested reader, the proofs of all the lemmas and theorems presented in this paper can be found in our technical report [20].

\section{RELATED WORK}

In this section, we briefly survey related work on competition and spectrum pricing for secondary markets and highlight the differing contributions of our paper. 
Network providers in spectrum markets may face competition at two different levels. The first level consists of competition between secondary network providers to lease spectrum from a primary provider (or the government) that holds a spectrum license. The second level of competition is between network providers holding a spectrum license or lease and competing to offer their services to end-users.

Many papers in the literature consider the first level of competition, while our paper addresses the second one. For instance, in the works by Jagannathan [16], Kasbekar [19], Duan [12], Ren [35], Niyato and Hossain [31], Sengupta and Chatterjee [37] and Xing [40], game theoretic approaches to spectrum auctioning and leasing are analyzed. The set-up of all these papers (i.e., competition between providers to lease spectrum) is different from ours (i.e., competition between providers to lure users).

Several papers study the problem of ensuring profitability in secondary spectrum markets. Niyato and Hossain [31] derives market equilibria pricing by taking into consideration the demand and supply dynamics of spectrum auctions. However, the model uses a very specific secondary demand based on the utility from owning the spectrum and how much it costs to lease the spectrum. Also, secondary users have the option to lease parts of their spectrum from different spectrum owners. On the end-user side, Alanyali et al. [6] establishes a pricing policy which guarantees profitability for the network provider as long as a demand is generated. However, this paper assumes a monopolistic framework, while ours considers an oligopolistic one. Furthermore, [6] considers a multi-cell setting with a single frequency band in each cell, while our paper focuses on an isolated cell offering multiple frequency bands.

Mutlu et al. [29] also consider a monopolistic framework and derive an optimal coordinated access policy under which revenue from secondary users is maximized. The results of that paper show that a threshold policy is optimal for coordinated access in an isolated cell, assuming that a provider advertises a fixed price (i.e., the price does not depend on the instantaneous channel occupancy). These results are leveraged for the analysis in our paper.

In the work by Ileri et al. [15], a comprehensive model including both the auction and the end-user sides of the competition is studied. Different from our paper, this model focuses on the auctioning side of the competition where the revenue generated by secondary users is used to compensate for the costs of auctioning. In our model, we assume that providers own spectrum and need only to consider the revenue brought in by the primary and secondary users.

The works by Maille and Tuffin [24] and Maille et al. [25] use a model where both the auction side and the service side of the competition are considered. The work in [24] specifically focuses on the competition between two different but substitute technologies while [25] models a three level competition, where spectrum owners, lessees and users each make their own separate decisions. These decisions include the use of different technologies. In our model, we assume that providers offer the same type of services and therefore cannot influence the secondary users' preferences beside the price advertised. A related work by Ren et al. [35] studies and compare the market outcome achieved by respectively enforcing cooperation or competition among providers. While such external interventions might be useful in analyzing hypothetical outcomes, our model refrains from such enforcements as it aims to characterize a natural competition. In a work by Kim et al. [21], competition between two providers is analyzed where network preemption allows for primary users to evict secondary users from the system. Unlike our paper, the network model is not a finite capacity multichannel network but rather a spatial distribution of channels that turn on and off, and the analysis relies on an approximation. In one recent study by Korcak et. al. [23], the possibility of collusion between several wireless network providers is considered. This collusion is based on a coalition game model. In contrast, in our model, network providers do not communicate with each other about their intentions (i.e., it is a non-cooperative game). Thus, the possibility of market sharing between the providers is purely a result of market dynamics.

None of the previous work surveyed here considers competition among network providers implementing optimal coordinated access and facing secondary demand governed by a general demand function. The characterization of the market equilibrium and demonstration of a price war won by the provider(s) with the lowest breakeven price as well as the possibility of market sharing equilibria under uncoordinated access policies are unique contributions of our paper.

\section{NETWORK MODEL}

In this section we introduce the considered model and notation. For convenience of exposition we present here a model with two providers, and later extend it to an arbitrary number of competing providers: Each provider $i=1,2$ has a finite $C_{i}$ number of channels, and a dedicated primary-user base whose intensity is represented with $\lambda_{i}>0$. For each primary user serviced, provider $i$ collects a reward of $K_{i}$ units.

The providers compete for an additional secondary 




Fig. 2. Two providers each with a capacity $C_{i}, i=1,2$, compete for secondary demand $\sigma(p)$ while also serving their respective dedicated (primary) demand $\lambda_{i}$

demand whose intensity is modulated by the prices they charge for secondary access. If provider $i$ charges $p_{i}$ units per secondary access then the intensity of secondary demand is $\sigma\left(\min \left\{p_{1}, p_{2}\right\}\right)$. Here $\sigma(\cdot)$ is the demand function and it is assumed to be continuous and non-increasing.

We shall assume that each demand type (primary and secondary) consists of a random sequence of request arrivals that occur according to independent Poisson processes, and intensity of demand represents the average number of requests per unit time. We also assume that, if granted, each request holds one channel for a random duration that is generally distributed with unit mean, independently of other requests and arrival times. We shall assume that the channel holding statistics are identical for primary and secondary requests. Such an assumption is valid when both types of traffic are generated by similar applications.

It is assumed that secondary demand is attracted to the provider which charges the lowest price. In the case when both providers charge the same price, the resulting secondary demand splits between the two providers according to a probability vector $\left[\alpha_{1}, \alpha_{2}\right]$ such that $\alpha_{1}+\alpha_{2}=1$ and $\alpha_{i}>0$. Namely, each provider $i$ receives secondary demand intensity $\alpha_{i} \sigma\left(p_{i}\right)$.

Each provider $i$ also has the choice of admitting or rejecting secondary requests according to an access policy, which we denote by $A_{i}$. We assume that actions taken by $A_{i}$ depend only on the total number of users (primary and secondary) in the system. Thus, $A_{i}$ belongs to the class of occupancy-based policies, which performance are insensitive to the call length distribution except through the mean [30].

Since demand is random and providers have a finite number of channels to provide service with, they cannot accommodate new requests if all of the channels are occupied. This results in some requests being blocked. We define $B_{i, j}\left(\lambda_{i}, \sigma, A_{i}\right)$ as the blocking probability for class $j$ users ( $j=1$ for primary and 2 for secondary) when secondary demand is $\sigma$ and the access policy is $A_{i}$.

The goal of each provider is to maximize the total revenue collected. The revenue rate of provider $i$ when it services secondary demand of $\sigma$ units is given by:

$$
\begin{aligned}
W_{i}\left(p_{i}, \sigma, A_{i}\right)= & \left(1-B_{i, 2}\left(\lambda_{i}, \sigma, A_{i}\right)\right) \sigma p_{i} \\
& +\left(1-B_{i, 1}\left(\lambda_{i}, \sigma, A_{i}\right)\right) \lambda_{i} K_{i} .
\end{aligned}
$$

Here the first and the second terms are respectively the revenue generated by primary and secondary requests that are admitted by the provider. Each term represents the expected long time rates per unit time.

Since the secondary demand a provider receives depends on prices of both providers, so does the revenue of the provider. We define the reward $R_{i}\left(p_{i}, p_{-i}\right)$ of provider $i$ as its revenue when provider $i$ and its competitor $-i$ charge secondary access $p_{i}$ and $p_{-i}$ units respectively. Namely,

$$
R_{i}\left(p_{i}, p_{i}\right)= \begin{cases}W_{i}\left(p_{i}, \sigma\left(p_{i}\right), A_{i}\right) & \text { if } p_{i}<p_{-i} \\ W_{i}\left(p_{i}, \alpha_{i} \sigma\left(p_{i}\right), A_{i}\right) & \text { if } p_{i}=p_{-i} \\ W_{i}\left(p_{i}, 0, A_{i}\right) & \text { if } p_{i}>p_{-i}\end{cases}
$$

Hence the reward is affected by the amount of secondary demand provider $i$ captures through the relationship between its own price $p_{i}$ and the price other provider chooses $p_{-i}$. Once the prices determine the secondary demand for each provider, the rewards are further shaped by the providers' access policies. Each provider has full information on its own network parameters and can observe the prices advertised by its competitors.

\section{Optimal CoOrdinated ACCess Policies And PROFITABILITY}

For a given secondary demand $\sigma$ and secondary price $p$, let $A_{i}^{*}(p, \sigma)$ denote a coordinated access policy that maximizes the revenue rate for provider $i$. We refer to $A_{i}^{*}(p, \sigma)$ as the optimal coordinated access policy. We represent the resulting maximal revenue $W_{i}^{*}(p, \sigma)$ as follows:

$$
\begin{aligned}
W_{i}^{*}(p, \sigma) & =W_{i}\left(p, \sigma, A_{i}^{*}(p, \sigma)\right) \\
& =\max _{A_{i}} W_{i}\left(p, \sigma, A_{i}\right)
\end{aligned}
$$

Under the given assumptions, it is well-known that the optimal coordinated access policy is a threshold (reservation) policy: Secondary users are admitted by a provider when the channel occupancy of the provider is 


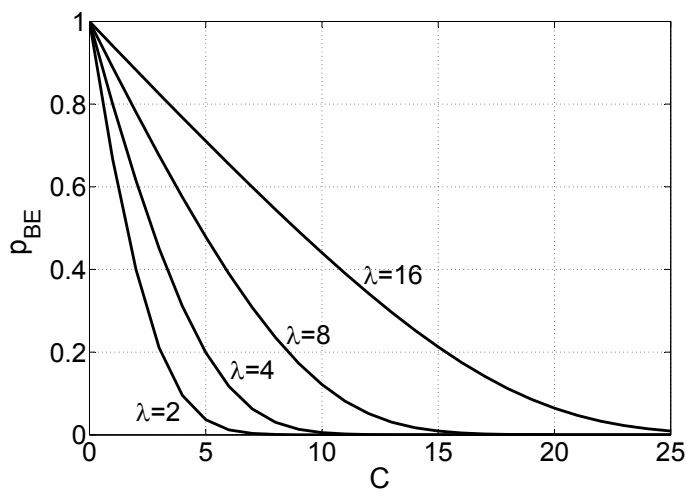

(a) Break-even price $p^{B E}$ with respect to network capacity $C$ for different primary arrival rates $\lambda$ and primary price $K=1$.

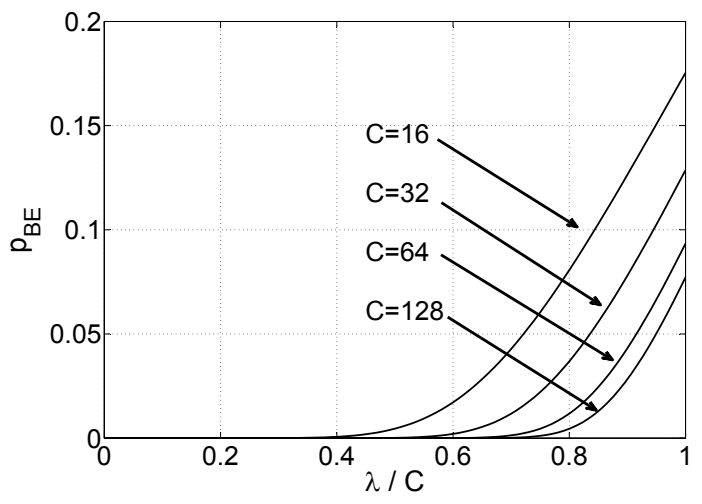

(b) Break-even price $p^{B E}$ with respect to network load $\lambda / C$ for different network capacities $C$ and primary price $K=1$.

Fig. 3. Behavior of break-even price as a function of network parameters.

below a threshold and they are blocked otherwise [22, $28,30,34]$. The optimal threshold value depends on all parameters of the provider including intensity of the secondary demand.

In the competitive setting considered in this paper it will be important to identify conditions under which an optimal policy $A_{i}^{*}(p, \sigma)$ ever accepts a secondary request. Under such conditions the secondary pricedemand pair $(p, \sigma)$ yields profit relative to serving primary demand only; in turn $(p, \sigma)$ represents an economically viable situation for provider $i$. The issue is closely related with the opportunity cost of accepting a secondary request: On the one hand such a request brings an immediate revenue of $p$, on the other hand it may cause rejecting future requests, possibly with higher immediate revenue, due to the channel that it holds temporally. It turns out that the sign of the balance in this tradeoff can be deduced by the so-called policy improvement method of dynamic programming technique [5,22]. Simply put, we can formulate a infinite horizon reward problem where we do not admit any secondary users. From this problem we can formulate the average reward that a primary user brings from a single admission to the network, which in turn yields the minimum reward $p_{i}^{B E}$ secondary users need to compensate for to gain admission to the network [20]. The analysis reveals that $p_{i}^{B E}$ can be expressed as follows:

$$
p_{i}^{B E}=K_{i} E\left(\lambda_{i}, C_{i}\right),
$$

where $E\left(\lambda_{i}, C_{i}\right)$ is the Erlang-B blocking formula given by

$$
E\left(\lambda_{i}, C\right)=\frac{\frac{\lambda_{i}^{C}}{C !}}{\sum_{k=1}^{C} \frac{\lambda_{i}^{k}}{k !}} .
$$

This leads to the following conclusion:
Theorem IV.1 For $\sigma>0$ :

(a) $W_{i}^{*}(p, \sigma)>W_{i}^{*}(p, 0)$ if $p>p_{i}^{B E}$,

(b) $W_{i}^{*}(p, \sigma)=W_{i}^{*}(p, 0)$ if $p \leq p_{i}^{B E}$.

Theorem IV.1(a) states that if the price exceeds $p_{i}^{B E}$ then serving secondary demand yields strictly higher revenue for provider $i$ than not serving it. Conversely, part (b) of the theorem states that secondary demand does not lead to any revenue improvement otherwise, implying that rejecting the entire secondary demand is optimal for such prices. In effect, at $p_{i}^{B E}$ the immediate revenue balances the opportunity cost of a secondary request. We therefore coin $p_{i}^{B E}$ as the break-even price for provider $i$.

It is striking that the break-even price expression (3) does not depend on the secondary demand. Namely, any price above $p_{i}^{B E}$ strictly improves the revenue of provider $i$ regardless of how much secondary demand it generates.

Figures 3(a) and 3(b) illustrate how the normalized break-even price (i.e., $p^{B E} / K$ ) changes with respect to relevant network parameters, namely the system capacity $C$ and the primary load $\lambda$. The normalized price is given by the Erlang-B function, which has been well studied in teletraffic theory. In particular upper and lower bounds are obtained in $[14,18]$, and it is demonstrated in [17] that for a given arrival load $\lambda$, the Erlang-B function (hence, the break-even price) is a convex function of the capacity $C$, as can be observed from Figure 3(a). It is also worth noting that as the network capacity increases, the value of the break-even price at the critical load where $\lambda=C$ decreases as demonstrated in Figure 3(b). 
Figure 3(b) shows that for an over-provisioned network (in which primary load $\lambda$ is below the capacity $C$ by a significant margin), the break-even price is substantially lower than the primary price. We observe that for $C=16$ the normalized break-even price is negligible compared to the primary price for network loads below $40 \%$, a number close to the network utilization measurements reported in [3]. As the network capacity increases, it takes even higher network loads to observe the slightest increase in the break-even price, almost as high as $80 \%$ when the capacity is increased to $C=128$. This result suggests that, in an over-provisioned network, spectrum sharing at secondary prices that are low relative to primary price would result in net profit, regardless of secondary demand.

\section{Market Equilibrium Under CoORdinAted SECONDARY ACCESS}

In competing for and serving secondary demand, a provider's action consists of an advertised price for secondary access and a local policy to coordinate secondary access. For any price, and for any demand the price raises, each provider's revenue is highest under optimal coordination. Hence optimal coordination is a dominating choice uniformly for all situations. In this section we will assume all providers implement optimal coordinated access. With this assumption each provider's strategic action reduces to a pricing decision.

We start with a characterization for competitive equilibria in the considered scenario. However, before we do that, it is important to recall the formal definition of a Nash equilibrium.

Definition V.1 A pricing strategy profile $\left(p_{1}, p_{2}\right)$ is a Nash equilibrium for rewards $R_{i}\left(p_{1}, p_{2}\right)$ if and only if

$$
\begin{aligned}
& R_{1}\left(p_{1}, p_{2}\right)=\max _{p} R_{1}\left(p, p_{2}\right) . \\
& R_{2}\left(p_{1}, p_{2}\right)=\max _{p} R_{2}\left(p_{1}, p\right) .
\end{aligned}
$$

The following theorem identifies possible market outcomes in terms of Nash equilibria. The first part of the theorem concerns the case when one provider (without loss of generality provider 1) has strictly lower breakeven price than the other provider. Then, in equilibrium, that provider sets its price below its competitor's breakeven price, and thereby captures the entire secondary demand irrespective of its shape. The second part of the theorem concerns the symmetric case when both providers have the same break-even price. It indicates that, in that case, providers are forced to share the secondary demand at their break-even prices and therefore make no profit.
Theorem V.1 (a) If $p_{1}^{B E}<p_{2}^{B E}$ then one or more Nash equilibria exist and have the form $\left(p_{1}, p_{2}\right)$ with

$$
\begin{gathered}
p_{1} \in \underset{p \in\left[p_{1}^{B E}, p_{2}^{B E}-\epsilon\right]}{\operatorname{argmax}}, W_{1}^{*}(p, \sigma(p)) \\
p_{2} \geq p_{2}^{B E},
\end{gathered}
$$

where $\epsilon$ is a sufficiently small discretization constant.

(b) If $p_{1}^{B E}=p_{2}^{B E}$ then there exists a unique Nash Equilibrium $\left(p_{1}, p_{2}\right)$ such that

$$
p_{1}=p_{2}=p_{1}^{B E} \text {. }
$$

Theorem V.1 states that if the break-even prices are different, the provider with the lower break-even price captures the entire market by pricing below its competitor's break-even price. However, when the price is continuous, it is impossible to provide an exact price value that achieves this best response. Hence, following a well-known approach used in game theory to address this technicality [33, pages 64-67], we assume that each provider's price is a multiple of a small discretization step $\epsilon$.

In the rest of this section we comment of various aspects of the equilibria described in Theorem V.1.

a) Examples: The following two examples aim to illustrate that qualitative differences in the placement of Nash equilibria are governed by the secondary demand function $\sigma(p)$. These examples are based on demand functions commonly used in the economics literature that are respectively exponentially and linearly decreasing with price [38].

Example V.1 Suppose that the secondary demand function follows a negative exponential demand given by:

$$
\sigma(p)=10 e^{-0.2 p},
$$

which indicates sufficiently low-elastic demand so that the revenue rate remains increasing with price. We set the network parameters for both providers as:

$$
\begin{aligned}
& \left(\lambda_{1}, C_{1}, K_{1}\right)=(1,2,20) \\
& \left(\lambda_{2}, C_{2}, K_{2}\right)=(10,5,35),
\end{aligned}
$$

which, through Eq. (3), yield

$$
\begin{aligned}
& p_{1}^{B E}=4.00, \\
& p_{2}^{B E}=19.74 .
\end{aligned}
$$

Figure 4(a) demonstrates the low-elasticity property of provider 1's revenue rate function, $W_{1}^{*}\left(p_{1}, \sigma\left(p_{1}\right)\right)$. The revenue rate is clearly maximized when the price is $p_{1}=$ $19.74-\epsilon$, at a price slightly below the other provider's break-even price. 


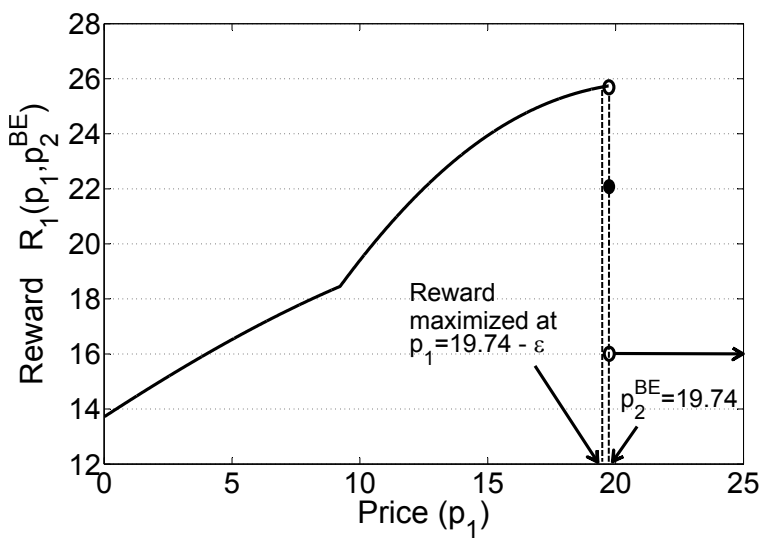

(a) Provider 1's reward maximized just below competitor's breakeven price when secondary demand is $\sigma\left(p_{1}\right)=10 e^{-0.2 p_{1}}$.

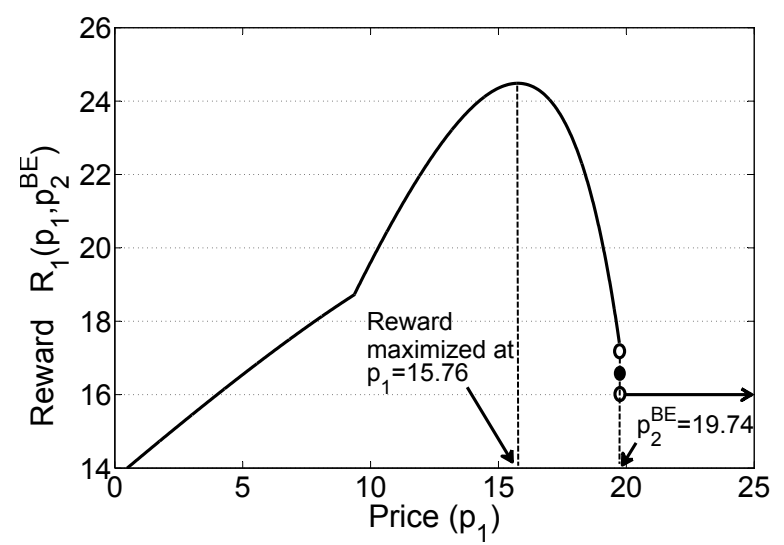

(b) Provider 1's reward maximized below competitor's break-even price when secondary demand is $\sigma\left(p_{1}\right)=10-0.5 p_{1}$.

Fig. 4. Different reward maximizing prices as provided in Examples V.1 and V.2.

Example V.2 In this example we consider a linear demand function given by:

$$
\sigma(p)=10-0.5 p
$$

The network parameters and thus the break-even price are the same as in the previous example, which we omit. Under this new and faster decreasing demand function, we plot the revenue rate in Figure 4(b). The revenue rate achieves its maximum at $p_{1}=15.76$ after which it demonstrates high-elasticity and starts to decrease with price. This results in the revenue maximizing price being less than $p_{2}^{B E}=19.74$. Therefore, facing such demand provider 1 would lower its price further below even though its competitor cannot match it without incurring a net loss, which demonstrates our result stated in Theorem V.1(b).

b) Interpretation of Nash equilibria in the asymmetric case: Without loss of generality let us consider the case $p_{1}^{B E}<p_{2}^{B E}$. Exact value of the equilibrium price $p_{1}$ depends on where provider 1's revenue is maximized on the interval $\left[p_{1}^{B E}, p_{2}^{B E}-\epsilon\right]$. Note that the revenue may attain its maximum at several price values of this interval, hence the set notation provided in the Theorem.

If the demand function $\sigma(p)$ results in the revenue rate being maximized right below the competitor's breakeven price such that:

$$
\underset{p \in\left[p_{1}^{B E}, p_{2}^{B E}-\epsilon\right]}{\arg \max } W_{1}^{*}(p, \sigma(p))=p_{2}^{B E}-\epsilon,
$$

then the price profile $\left(p_{2}^{B E}-\epsilon, p_{2}^{B E}\right)$ is the Nash equilibrium and no other equilibrium exists. This property holds, for example, when $\sigma(p)$ is constant and thus demand is inelastic.

The equilibrium price of provider 2 is strictly above its break-even price $\left(p_{2}>p_{2}^{B E}\right)$ if all maxima of provider 1's revenue rate occur within the subinterval $\left[p_{1}^{B E}, p_{2}^{B E}-\epsilon\right]$. Only then provider 2 can choose to advertise a higher price without creating an incentive for provider 1 to deviate to a revenue maximizing price that is above provider 2's break-even price. Hence while all Nash equilibria have the form given in Theorem V.1(a), not any price profile that has this form is necessarily a Nash equilibrium.

c) Profitability: Provider $i$ profits from providing secondary access only if it's break-even price is lower than its competitor's (i.e., $p_{i}^{B E}<p_{-i}^{B E}$ ): In this case the provider $i$ 's payoff given by $R_{i}\left(p_{-i}^{B E}-\epsilon, p_{-i}^{B E}\right)=$ $W_{i}^{*}\left(p_{-i}^{B E}-\epsilon, \sigma\left(p_{-i}^{B E}\right)\right)$ strictly exceeds the revenue without secondary demand $W_{i}^{*}(\cdot, 0)$; hence profitability of provider $i$ follows. Otherwise provider $i$ does not profit from secondary demand: If $p_{i}^{B E}>p_{-i}^{B E}$ it serves no secondary users and if $p_{i}^{B E}=p_{-i}^{B E}$ it serves secondary users exactly at its break-even price. We point out that in the latter symmetric case neither provider achieves positive profit.

d) Best response dynamics: The equilibria provided in Theorem V.1 are consequences of best response dynamics. The best response of provider $i$ when facing a competitor price $p_{-i}$ is to set its price slightly lower in order to capture all of the secondary demand. This can be formalized using the following lemma.

Lemma V.1 If $p_{i}>p_{i}^{B E}$, then there is a price $p_{i}^{\prime}$ such 
that $p_{i}^{B E}<p_{i}^{\prime}<p_{i}$ and

$$
W_{i}^{*}\left(p_{i}^{\prime}, \sigma\left(p_{i}^{\prime}\right)\right)>W_{i}^{*}\left(p_{i}, \alpha_{i} \sigma\left(p_{i}\right)\right) .
$$

Namely, if provider $i$ profits at a given price, obtaining the entire secondary demand is strictly more profitable than obtaining part of the demand at a slightly higher price. This property reflects an incentive for each provider to unilaterally deviate from offering the same price as its opponent, provided that the price is strictly above its break-even price. A formal proof of Theorem $\mathrm{V} .1$ is given in [20] by making use of this best response.

Given initial prices $p_{1}$ and $p_{2}$ such that $p_{i}>p_{i}^{B E}, i=$ 1,2 , both providers will follow this strategy, lowering their prices in turn. This process continues until the market price drops so low that the provider with the higher break-even price finds himself unable to lower its price any further without incurring a net loss. Then the other provider will choose to match this price or lower its price slightly below, depending on what its own breakeven price is in comparison.

e) Comparison with classical Bertrand duopoly:

Theorem V.1 essentially asserts that the equilibrium outcome of competition for secondary demand is a price war. Price wars are also typical outcomes in the classical Bertrand duopoly, hence it is worthwhile to put the two settings in perspective. In the Bertrand game, for a given price, both the revenue and the cost are linear functions of demand. In contrast, in the present setting neither revenue nor cost of secondary service is linear in secondary demand, primarily because both quantities rely heavily on blocking probabilities that are highly nonlinear in the demand. In addition, the Bertrand model precludes any capacity constraints and assumes that all demand can be satisfied, whereas the model of this paper is centered on a fundamental limitation in capacity. Yet, interestingly, the equilibrium of the present game resembles (and, depending on the secondary demand function, may be identical to) the outcome of a Bertrand game in which marginal cost is constant and equal to the break-even price.

This similarity is a consequence of two nontrivial properties established in the present paper: $(i)$ insensitivity of break-even price against secondary demand, and ( $i$ i) Lemma V.1, which indicates that having more secondary demand is always more favorable provided that secondary service is priced above break-even price. Both properties, however, rely on the assumption of optimal coordination of secondary access and may not extend to arbitrary access policies. In particular the following section establishes that in the specific case of uncoordinated secondary access, competitive market equilibria may reflect market sharing at a continuum of prices that are strictly above break-even prices, presenting a drastic deviation from Bertrand duopoly.

f) Extension to multiple providers: Equilibrium descriptions of Theorem V.1 can be generalized to an arbitrary number of providers competing for the secondary demand, each with their own primary users, capacities and primary user rewards: Consider $N$ such providers and let $p_{i}^{B E}$ continue to represent the break-even price of provider $i$. Without any loss of generality, let us reindex the providers if necessary so that: $p_{1}^{B E} \leq p_{2}^{B E} \leq$ $p_{3}^{B E} \leq \ldots \leq p_{N}^{B E}$.

Further we define:

$$
n=\max \left\{i: p_{i}^{B E}=p_{1}^{B E}\right\} .
$$

Hence $n$ is the number of providers that have the smallest break-even price. We generalize the two cases presented in Theorem V.1 as follows:

- If $n>1$ then any price profile $\left(p_{1}, p_{2}, \cdots, p_{N}\right)$ such that

$$
\begin{gathered}
p_{i}=p_{1}^{B E} \quad \text { for } i=1,2, \cdots, n \\
p_{i}>p_{1}^{B E} \text { for } i=n+1, n+2, \cdots, N .
\end{gathered}
$$

is an Nash equilibrium. In each such equilibrium providers $1,2, \cdots, n$ service the secondary demand at their break-even prices thereby generating no additional profit. The secondary demand is split among these providers according to an arbitrary probability vector $\left[\alpha_{1}, \alpha_{2}, \ldots, \alpha_{n-1}, \alpha_{n}\right]$ where $\sum_{i}^{n} \alpha_{i}=1, \alpha_{i}>0$, which has no bearing on equilibrium prices. The remaining $N-n$ providers do not capture any secondary demand.

- If $n=1$ then there is a single provider whose breakeven price is lower than all the rest. In equilibrium this provider captures the entire secondary demand at a strictly profitable price, while the remaining $N-1$ providers cannot serve any secondary demand. In particular Nash equilibria have the form

$$
\begin{aligned}
& p_{1} \in \underset{p \in\left[p_{1}^{B E}, p_{2}^{B E}-\epsilon\right]}{\operatorname{argmax}} W_{1}^{*}(p, \sigma(p)), \\
& p_{i} \geq p_{2}^{B E} \text { for } i=2,3, \cdots, N .
\end{aligned}
$$

\section{MARKET EQUILIBRIUM UNDER UNCOORDINATED SECONDARY ACCESS}

In this section we consider equilibrium regimes that arise when competing providers grant uncoordinated access to secondary demand. We shall argue that such equilibria can be characterized explicitly and may be drastically different than those under coordinated access. In order to facilitate the exposition, this section focuses on inelastic demand (that is, $\sigma(p)=\sigma$ ) but its qualitative conclusions extend to general demand functions. 
Under uncoordinated access, a provider does not differentiate between primary and secondary users in granting spectrum access requests. In turn, both types of users experience the same blocking probability. This probability depends on the aggregate demand and system capacity, and can be computed using standard techniques in teletraffic. Namely, when provider $i$ serves secondary demand $\sigma$, the two blocking probabilities are

$$
B_{i, 2}\left(\lambda_{i}, \sigma, A_{i}\right)=B_{i, 1}\left(\lambda_{i}, \sigma, A_{i}\right)=E\left(\lambda_{i}+\sigma, C_{i}\right),
$$

where $E\left(\lambda_{i}+\sigma, C\right)$ is the Erlang-B formula given by (4).

The revenue rate of provider $i$, when serving secondary demand $\sigma$ by charging $p_{i}$ per admitted request, is then given by

$$
\begin{aligned}
\hat{W}_{i}\left(p_{i}, \sigma\right)= & \left(1-E\left(\lambda_{i}+\sigma, C_{i}\right)\right) \sigma p_{i} \\
& +\left(1-E\left(\lambda_{i}+\sigma, C_{i}\right)\right) \lambda_{i} K_{i},
\end{aligned}
$$

where the first term corresponds to the reward collected from secondary users that gain admission to the network, while the second term corresponds to the reward collected from the serviced primary users. (Here and in the rest of this section we will consistently use the symbol to indicate the quantities associated with uncoordinated access.)

\section{A. Profitability}

We recognize $\hat{W}_{i}\left(p_{i}, 0\right)$ as the revenue rate of provider $i$ when it does not serve any secondary demand. Similar to the profitability conditions for the optimal coordinated access case stated in Theorem IV.1, note that

$$
\hat{W}_{i}\left(p_{i}, \sigma\right) \geq \hat{W}_{i}\left(p_{i}, 0\right)
$$

if and only if $p_{i} \geq \hat{p}_{i}^{B E}$ where

$$
\hat{p}_{i}^{B E}=\frac{\left(E\left(\lambda_{i}+\sigma, C_{i}\right)-E\left(\lambda_{i}, C_{i}\right)\right) \lambda_{i} K_{i}}{\left(1-E\left(\lambda_{i}+\sigma, C_{i}\right)\right) \sigma} .
$$

Hence the provider incurs loss and has no incentive to serve the secondary demand at a price below $\hat{p}_{i}^{B E}$. In turn $\hat{p}_{i}^{B E}$ is the break-even price of provider $i$ under uncoordinated access.

It is instructive to compare the break-even prices under uncoordinated access and optimal coordinated access. Firstly, $\hat{p}_{i}^{B E} \geq p_{i}^{B E}$ because if the optimal admission policy does not yield positive profit from secondary demand then neither does any other policy. For typical parameters this inequality is strict. Consequently, providers need to charge a higher price to secondary users in order to avoid a net loss, which results in the tendency to bid higher prices under uncoordinated access. Secondly, in contrast to $p_{i}^{B E}$, the break-even price $\hat{p}_{i}^{B E}$ for uncoordinated access depends on the value of secondary demand $\sigma$.

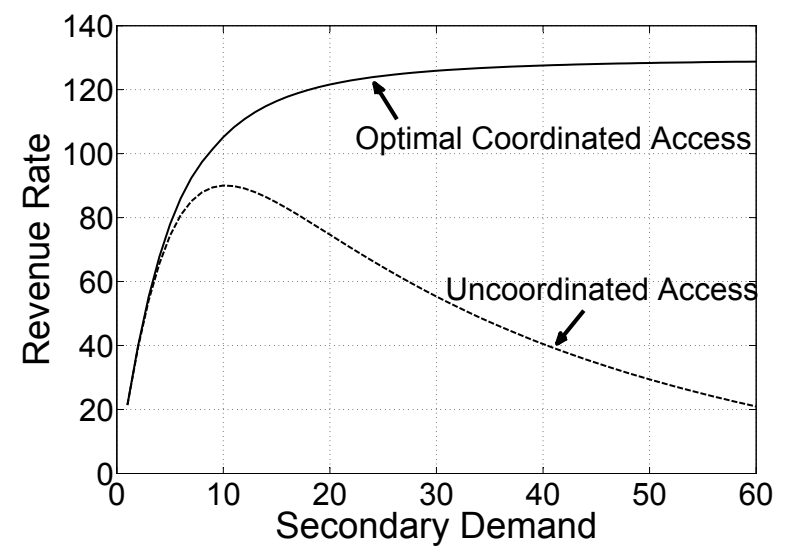

Fig. 5. Revenue rates under optimal coordinated and uncoordinated access versus secondary demand - network parameters: $p_{i}=30, \lambda_{i}=$ $13, C_{i}=20$ and $K_{i}=50$.

\section{B. Market Sharing}

While Lemma V.1 establishes that market sharing is not favorable under optimal coordinated access, these results do not necessarily extend to a case when uncoordinated access is implemented. As it turns out, under an uncoordinated access implementation, whether the revenue rate increases or decreases by sharing secondary demand depends on another critical parameter we shall establish later.

Before we get into our analysis, it is insightful to compare how the revenue rates $\hat{W}_{i}\left(p_{i}, \sigma\right)$ and $W_{i}^{*}\left(p_{i}, \sigma\right)$ behave under uncoordinated and optimal coordinated access strategies. Figure 5 illustrates the two revenue rates for a range of secondary demand $\sigma$, when all other parameters are fixed. When plotting both revenue rates, the secondary price $p_{i}$ is chosen above both break-even prices so that the optimal revenue rate $W_{i}^{*}\left(p_{i}, \sigma\right)$ is strictly increasing in $\sigma$. As a by-product of optimality, $W_{i}^{*}\left(p_{i}, \sigma\right) \geq \hat{W}_{i}\left(p_{i}, \sigma\right)$ under all circumstances.

However, $\hat{W}_{i}\left(p_{i}, \sigma\right)$ has an important qualitative difference relative to its optimal counterpart: It increases for a range of secondary demand $\sigma$ and decreases afterwards. This happens because for small $\sigma$, secondary users enhance revenue by using the leftover capacity from primary users, but as $\sigma$ increases secondary access occurs at an increasing expense of primary access and that leads to a decline in revenue if primary users are more valuable (that is, if $p_{i}<K_{i}$ ). This property opens the possibility that $\hat{W}_{i}\left(p_{i}, \alpha_{i} \sigma\right)>\hat{W}_{i}\left(p_{i}, \sigma\right)$, in which case a provider has incentive to share secondary demand at prices higher than break-even. Consequently, it has a profound impact on the outcome of a competitive setting. 


\begin{tabular}{|c||c|c|}
\hline Access Policy & Equilibrium price & Equilibrium profit \\
\hline Coordinated & $p_{1}=p_{2}=0.91$ & $P_{1}=P_{2}=0$ \\
Uncoordinated & $23.46 \leq \hat{p}_{1}=\hat{p}_{2} \leq 34.11$ & $0 \leq \hat{P}_{1}=\hat{P}_{2} \leq 121.54$ \\
\hline
\end{tabular}

TABLE I

EQUILIBRIUM PRICES AND RESULTING PROFITS FOR THE SETTING CONSIDERED IN EXAMPLE VI.1.

To formalize this intuition let us define

$$
\bar{p}_{i}=\frac{\left(E\left(\lambda_{i}+\sigma, C_{i}\right)-E\left(\lambda_{i}+\alpha_{i} \sigma, C_{i}\right)\right) \lambda_{i} K_{i}}{\left(1-E\left(\lambda_{i}+\alpha_{i} \sigma\right)\right) \alpha_{i} \sigma-\left(1-E\left(\lambda_{i}+\sigma\right)\right) \sigma} .
$$

It can be verified directly from (6) that:

$$
\begin{aligned}
& \hat{W}_{i}\left(p_{i}, \alpha_{i} \sigma\right)>\hat{W}_{i}\left(p_{i}, \sigma\right) \text { for } p_{i}<\bar{p}_{i} \\
& \hat{W}_{i}\left(p_{i}, \alpha_{i} \sigma\right) \leq \hat{W}_{i}\left(p_{i}, \sigma\right) \text { for } p_{i} \geq \bar{p}_{i} .
\end{aligned}
$$

Thus, only up to the price value $\bar{p}_{i}$, any provider $i$ would benefit from a reduction in its secondary demand. The price $\bar{p}_{i}$ can be interpreted as a market sharing threshold for provider $i$ : Any price above this threshold renders secondary demand too valuable to share and warrants a price war. Below this threshold, the provider has an incentive to remain at an equilibrium that reflects market sharing, provided the price satisfies the initial profitability condition in Eq. (7), which translates to being above the break-even price $\hat{p}_{i}^{B E}$.

\section{Equilibrium}

Competitive equilibria under uncoordinated access can now be determined depending on the critical price values $\hat{p}_{i}^{B E}$ and $\bar{p}_{i}$ of all providers $i$. Figure 6 illustrates a particular placement of these parameters for two providers. In the illustrated setting, the market sharing threshold exceeds the break-even price (that is, $\bar{p}_{i}>\hat{p}_{i}^{B E}$ ) for each provider. Furthermore the price intervals $\left[\hat{p}_{1}^{B E}, \bar{p}_{1}\right]$ and $\left[\hat{p}_{2}^{B E}, \bar{p}_{2}\right]$ have a non-empty intersection; therefore there exist common price values that are above breakeven values and acceptable for market sharing for both providers. In turn, there is a continuum of equilibria strictly above the break-even prices. We point out that the nature of equilibria depend on the relative ordering of $\hat{p}_{i}^{B E}, \bar{p}_{i}$ of all providers.

We conclude this section with a numerical example concerning a symmetric setting.

Example VI.1 We consider two network providers with identical parameters: Primary arrival rate $\lambda_{i}=13$, capacity $C_{i}=20$, and revenue collected per serviced primary user $K_{i}=50$. We continue to assume inelastic secondary demand whose value is chosen to be $\sigma=20$. We assume that secondary demand splits equally in the case of equal prices, that is, $\alpha_{1}=\alpha_{2}=0.5$.

The break-even price for coordinated access is computed as 0.91 ; hence by Theorem V.1 the unique price

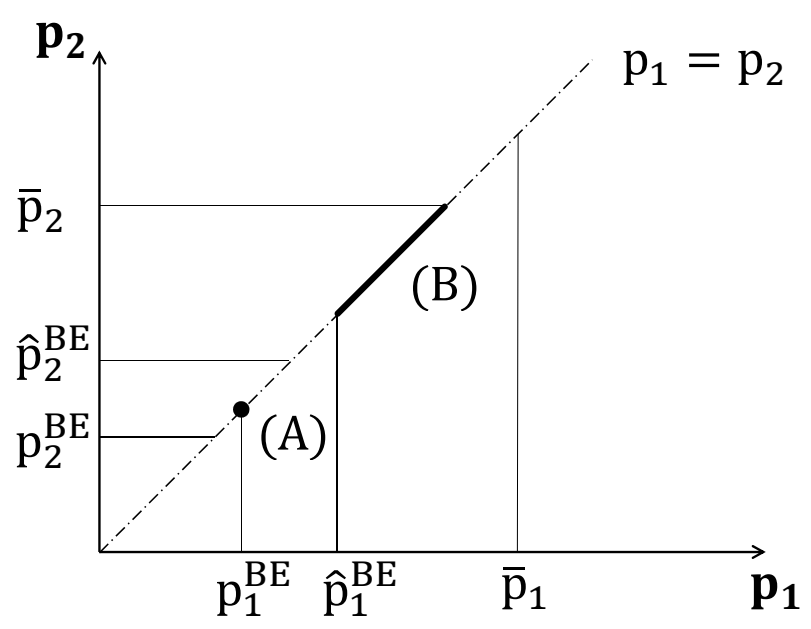

Fig. 6. Point (A) represents the Nash equilibrium under optimal coordinated access, and the continuum of points in (B) is the set of Nash equilibria under uncoordinated access.

equilibrium under coordinated access is $p_{1}=p_{2}=0.91$ and no provider profits from secondary demand.

The break-even price for uncoordinated access is $\hat{p}_{i}^{B E}=23.46$ and the market sharing threshold is $\bar{p}_{i}=34.11$. Hence, any price profile $(p, p)$ where $p$ lies in the interval $[23.46,34.11]$ constitutes a competitive equilibrium. For example, if provider $-i$ adopts the secondary price $p_{-i}=30$ then

$$
R_{i}\left(p_{i}, p_{-i}\right)= \begin{cases}\hat{W}_{i}\left(p_{i}, \sigma\right)=74.66 & \text { if } p_{i}=29.99 \\ \hat{W}_{i}\left(p_{i}, \frac{\sigma}{2}\right)=90.01 & \text { if } p_{i}=30 \\ \hat{W}_{i}\left(p_{i}, 0\right)=0 & \text { if } p_{i}>30\end{cases}
$$

in particular $p_{i}=30$ is the best response of provider $i$; and so the price profile $(30,30)$ is a Nash equilibrium. A comparison of possible equilibria and associated profits under both access strategies is given in Table I.

Interestingly, a policy (here uncoordinated access) that is sub-optimal for a provider in isolation results in competitive equilibria in which all providers are strictly better off than resorting to their optimal individual policies.

\section{CONCLUSION}

In this paper we investigated the equilibrium outcomes in a secondary spectrum market where multiple firms compete against each other. We focused on 
two proposed regimes for secondary spectrum access, namely coordinated access and uncoordinated access under private commons. Such an investigation can help provide important guidance to a firm's strategic decision process, by explicitly determining the parameters on which market success depends. To accomplish this goal, we formulated the problem as a non-cooperative game, in which providers with finite network capacities are making strategic pricing and access control decisions with respect to secondary users.

We first analyzed the case of coordinated access, for which the optimal access policy is of threshold type. We demonstrated that each provider has a unique break-even price, above which profitability is guaranteed regardless of the secondary user demand response. We provided an explicit analytical formula for the breakeven price, thus establishing its relationship with the system parameters (i.e., the primary load, primary reward, and system capacity). The break-even price of each provider is independent of the system parameters of other providers. Interestingly, the break-even price is, in general, significantly smaller than the primary reward. Thus, the break-even price is less than $1 \%$ of the primary reward if the primary load is below $68 \%$ and the number of channels $C$ exceeds 32 . Even at the critical load where the primary load is equal to the system capacity (i.e., $\lambda=C$ ), the break-even price remains below $20 \%$ of the primary reward for $C \geq 16$.

Next, using the notion of Nash equilibrium, we formalized the possible outcomes resulting from a noncooperative game in which optimal coordinated access is implemented by two or more network providers. We explained how the best response dynamics of each provider reflect a price war, in which each provider is driven into advertising a price slightly below that of its competitors as long as this price is above the breakeven price. This price war leads to a single provider (that with the lowest break-even price) capturing the entire secondary spectrum market. Although the demand function does not play a role in determining the identity of the winning provider, we showed that is does affects the revenue-maximizing price for that provider and the placement of the Nash equilibria. In the case of multiple providers sharing the same lowest break-even price, the game results in a market equilibrium in which none of the providers make profits.

Finally, we showed that the market dynamics fundamentally differ when providers implement uncoordinated access. We demonstrated that the break-even price is no longer insensitive to the secondary demand and market sharing becomes a possible best response. It is worth noting that even though a provider $i$ might find it desirable to share the market, it would still go into a price war for price values higher than its market sharing price $\bar{p}_{i}$, thus preventing convergence to an arbitrarily high price for secondary access. The possible market outcomes under an uncoordinated access policy become complex when the number of providers increases, but deserve further study, since they may result in a larger number of providers joining the market and higher revenues than possible under an optimal coordinated access policy.

\section{ACKNOWLEDGEMENTS}

This work was supported, in part, by the US National Science Foundation under grant CCF-0964652.

\section{REFERENCES}

[1] The Federal Communications Commission, Promoting Efficient Use of Spectrum Through Elimination of Barriers to the Development of Secondary Markets. http://hraunfoss.fcc.gov/edocs_ public/attachmatch/FCC-04-167A1.pdf

[2] Radio Spectrum Policy Group. http://rspg.groups.eu.int.

[3] Shared Spectrum Company, General Survey of Radio Frequency Bands (30 MHz to $3 \mathrm{GHz}$ ): Vienna, Virginia, September 1-5, 2009. http://www.sharedspectrum.com/wp-content/uploads/ 2010_0923-General-Band-Survey-30MHz-to-3GHz.pdf

[4] I.F. Akyildiz, W.Y. Lee, M.C. Vuran, and S. Mohanty. Next generation/dynamic spectrum access/cognitive radio wireless networks: a survey. Computer Networks, 50(13):2127-2159, 2006.

[5] A. Al Daoud, M. Alanyali, D. Starobinski. Profit-Robust Policies for Secondary Spectrum Sharing, Boston University CISE Tech. Report \# 2009-IR-0007, August, 2009.

[6] M. Alanyali, A. Al Daoud, D. Starobinski. Profitability of dynamic spectrum provision for secondary use. In DySPAN, May 2011.

[7] J. Bae, E. Beigman, R. Berry, M.L. Honig, H. Shen, R. Vohra, and H. Zhou. Spectrum markets for wireless services. In DySPAN, pages 1-10, Chicago, 14-17 October 2008.

[8] M.M. Buddhikot. Understanding dynamic spectrum access: Models, taxonomy and challenges. In DySPAN, pp.649-663, 2007.

[9] M. Bykowsky. A secondary market for the trading of spectrum: promoting market liquidity. Telecommunications Policy, 27(7):533 -541, 2003.

[10] M. Bykowsky, M. Olson, and W. Sharkey. Efficiency gains from using a market approach to spectrum management. Information Economics and Policy, 22(1):73-90, 2010.

[11] J.M. Chapin, and W.H. Lehr. The path to market success for dynamic spectrum access technology. IEEE Communications Magazine, 45(5):96-103, May 2007.

[12] L. Duan, J. Huang, and B. Shou. Competition with dynamic spectrum leasing. In CoRR, abs/1003.5517, 2010.

[13] Z. Han, C. Pandana, K.J.R. Liu. Distributive Opportunistic Spectrum Access for Cognitive Radio using Correlated Equilibrium and No-Regret Learning. Wireless Communications and Networking Conference, IEEE, March 2007.

[14] A. Harel. Sharp Bounds and Simple Approximations for the Erlang Delay and Loss Formulas. Management Science, Vol. 34, No. 8, August 1988, pp. 959-972.

[15] O. Ileri, D. Samardzija, and N.B. Mandayam. Demand responsive pricing and competitive spectrum allocation via a spectrum server. In DySPAN, pages 194-202, 8-11 2005.

[16] K. Jagannathan, I. Menache, E. Modiano, G. Zussman. Noncooperative Spectrum Access - The Dedicated vs. Free Spectrum Choice. In MobiHoc, May 2011.

[17] A.A Jagers, E. A. Van Doorn. On the continued Erlang loss function. Operations Research Letters, Vol. 5, No. 1, June 1986, pp. 43-46, 
[18] A. J. E. M. Janssen, J. S. H. Van Leeuwaarden, B. Zwart. Gaussian Expansions and Bounds for the Poisson Distribution Applied to the Erlang B Formula Advances in Applied Probability, Vol. 40, No. 1, March 2008, pp. 122-143.

[19] G.S. Kasbekar, S. Sarkar. Spectrum pricing games with arbitrary bandwidth availability probabilities. In ISIT, July 2011.

[20] E. Kavurmacioglu, M. Alanyali, D. Starobinski. Price Wars in Secondary Spectrum Markets: Competition or Collusion?, Boston University CISE Tech. Report \# 2012-IR-0001, January 2012. http://www.bu.edu/phpbin/cise/download.php?publication_ id $=1242$

[21] H. Kim, J. Choi, and K.G. Shin. Wi-Fi 2.0: Price and Quality Competitions of Duopoly Cognitive Radio Wireless Service Providers with Time-varying Spectrum Availability. In INFOCOM 2011.

[22] P. Key. Optimal control and trunk reservation in loss networks. Probability in the Engineering and Informational Sciences, 4, 1990, pp. 203-242.

[23] O. Korcak, T. Alpcan, and G. Iosifidis. Collusion of Operators in Wireless Spectrum Markets. In WiOpt 2012.

[24] P. Maille, B. Tuffin. Price war in heterogeneous wireless networks. Computer Networks, Volume 54, Issue 13, 15 September 2010, Pages 2281-2292.

[25] P. Maille, B. Tuffin, J.M. Vigne. Technological investment games among wireless telecommunications service providers. International Journal of Network Management, vol. 21, no. 1.

[26] J.W. Mayo, and S. Wallsten. Enabling efficient wireless communications: The role of secondary spectrum markets. Information Economics and Policy, 22(1):61-72, 2010.

[27] M. Michalopoulou, J. Riihijarvi, and P. Mahonen. Towards Characterizing Primary Usage in Cellular Networks: A Traffic-based Study. In DYSPAN, Aachen Germany, May 2011.

[28] B. Miller. A queueing reward system with several customer classes. Management Science, vol.16, pp. 234-245, 1971.

[29] H. Mutlu, M. Alanyali, and D. Starobinski. Spot pricing of secondary spectrum usage in wireless cellular networks. IEEE/ACM
Transactions on Networks, vol. 17, no. 6, pp. 1794-1804, December 2009.

[30] H. Mutlu, M. Alanyali, and D. Starobinski. On-line Pricing of Secondary Spectrum Access with Unknown Demand Function and Call Length Distribution. In INFOCOM 2010, March 2010.

[31] D. Niyato, E. Hossain. Competitive Pricing for Spectrum Sharing in Cognitive Radio Networks: Dynamic Game, Inefficiency of Nash Equilibrium, and Collusion. Selected Areas in Communications, IEEE Journal on , vol.26, no.1, pp.192-202, Jan. 2008

[32] T. Nguyen, H. Zhou, R.A. Berry, M.L. Honig, R. Vohra. The impact of additional unlicensed spectrum on wireless services competition. In DySPAN, May 2011.

[33] M. J. Osbourne. (2004) pp. 64-67 An Introduction to Game Theory. Oxford University Press, New York, NY, USA.

[34] R. Ramjee, D. Towsley, and R. Nagarajan. On optimal call admission control in cellular networks. Wireless Networks, vol. 3, pp. 29-41, 1997.

[35] Y. Ren, Q. Zhang, P. Chen. Cooperative and Non-cooperative WSPs in Dynamic Spectrum Leasing. In Communications and Mobile Computing pp. 274-277, 2011.

[36] J.B. Rosen. Existence and Uniqueness of Equilibrium Points for Concave N-Person Games. Econometrica, Vol. 33, No. 3, pp. 520-534, 1965.

[37] S. Sengupta, and M. Chatterjee. An economic framework for dynamic spectrum access and service pricing. Networking, IEEE/ACM Transactions on, 17(4):1200-1213, August 2009.

[38] K. T. Talluri, G. V. Ryzin (2004) pp. 321-323 The Theory and Practice of Revenue Management. Springer Science+Business Media, New York, NY, USA.

[39] C. Wang, H. Chen, X. Hong, M. Guizani. Cognitive radio network management. Vehicular Technology Magazine, IEEE , vol.3, no.1, pp.28-35, March 2008.

[40] Y. Xing, R. Chandramouli, and C. Cordeiro. Price dynamics in competitive agile spectrum access markets. Selected Areas in Communications, IEEE Journal on, 25(3):613-621, April 2007. 